\title{
Calidad de vida relacionada con la salud, la ansiedad y depresión en personas con cardiodesfibrilador
}

\author{
Quality of life related to health, anxiety and depression in people with cardioverter defibrillators \\ María Andreina Pulido-Montes ${ }^{*}$ orcid.org/0000-0002-3978-8574 \\ Luz Stella Bueno-Robles² orcid.org/0000-0002-9842-536X
}

1. Facultad de Enfermería, Universidad Cooperativa de Colombia. Bucaramanga, Colombia

2. Facultad de Enfermería, Universidad Nacional de Colombia. Bogotá, Colombia.

\section{Resumen}

Introducción: Las enfermedades cardiovasculares han provocado el incremento de arritmias en la población, aumentando el uso de dispositivos como el cardiodesfibrilador, que, si bien previene las arritmias, puede afectar la calidad de vida. Objetivo: Describir la asociación que existe entre la calidad de vida relacionada con la salud, la ansiedad y la depresión en personas portadoras de un cardiodesfibrilador. Materiales y métodos: Estudio cuantitativo, descriptivo correlacional, de corte transversal, donde participaron 85 pacientes con implante de cardiodesfibrilador. Se aplicó una ficha sociodemográfica, los instrumentos Cuestionario de Salud (SF-36), el Inventario de Ansiedad y Depresión de Beck. Resultados: El 81,2\% de los participantes fueron hombres con rango de edad 36-92 años. La calidad de vida relacionada con la salud mostró correlación negativa media con respecto a la ansiedad $(r=-0,622 ; p<0,001)$ y moderada, respecto a la depresión $(r s=-0,599 ; p<0,001)$. Conclusiones: Portar un cardiodesfibrilador puede desencadenar ansiedad y depresión, si estos no se intervienen oportunamente la calidad de vida relacionada con la salud puede deteriorarse, por ello, es importante implementar acciones tempranas que generen bienestar.

Palabras clave: Calidad de vida; ansiedad; depresión; enfermería cardiovascular, catéteres de permanencia; muerte súbita; desfibriladores implantables. (Fuente: DeCS, Bireme).

\begin{abstract}
Introduction: Cardiovascular diseases have caused a rise in arrhythmias, increasing the use caridoverter defibrillators. Although, these devices prevent arrhythmias, they may also affect the quality of life. Objective: To describe the association between quality of life and health, anxiety and depression in people who use cardioverter defibrillators. Materials and methods: A qualitative, descriptive, correlational and cross-sectional study was carried out with 85 patients using implantable cardioverter defibrillator. Sociodemographic records, the Health Questionnaire (SF-36), and the Beck Anxiety and Depression Questionnaire were used. Results: $81.2 \%$ of participants were men with their ages ranging between 36 and 92 years. The health-related quality of life showed a negative correlation with respect to anxiety ( $r=-0.622)$ and a slight correlation with depression ( $r s=-0.599 ; p<0.001)$. Conclusions: The use of a cardioverter-defibrillator can trigger anxiety and depression that can deteriorate the health-related quality of life if not timely intervened. It is important to implement early actions that generate well-being.
\end{abstract}

Keywords: Quality of life; anxiety; depression; cardiovascular nursing; catheters; indwelling; death; sudden; defibrillators, implantable. (Source: DeCS, Bireme).

\footnotetext{
*Autor de correspondencia

María Andreina Pulido-Montes

e-mail: mariaa.pulido@campusucc.edu.co
} 


\section{Introducción}

Según la Organización Mundial de la Salud (OMS), las enfermedades cardiovasculares, son la principal causa de muerte en el mundo, son responsables de 15,2 millones de defunciones en 2016 y han sido las principales causas de mortalidad durante los últimos 15 años(1).

En Colombia, el Observatorio Nacional de Salud (ONS) informó que en el año 2014 el total de fallecidos por enfermedad cardiaca isquémica ascendió a 32.338 por cada 100.000 habitantes, de acuerdo a lo anterior para el 2030 morirán cerca de 23.300.000 personas por esta patología(2).

Las personas con enfermedad cardíaca pueden ser portadoras de un cardiodesfibrilador, por prevención primaria o secundaria evitando la muerte súbita producida por arritmias letales. Este dispositivo que se implanta bajo la piel en el área superior del pecho, es capaz de detectar arritmias ventriculares letales, y proporciona descargas eléctricas para que el corazón regrese a su ritmo normal(3).

A nivel mundial, se ha estudiado poco sobre el número de pacientes con dispositivos cardiacos implantables(4). Sin embargo, se sabe que la tasa anual mundial de implantes ha aumentado a más de 100.000 unidades(5). En Estados Unidos, se implantan 300.000 nuevos dispositivos por año en pacientes con disfunción de ventrículo izquierdo y enfermedad coronaria(6). El implante del dispositivo expone a los pacientes a una situación de vulnerabilidad física y psicológica que puede afectar negativamente su calidad de vida relacionada con la salud(7). Estudios realizados en China y México demuestran que la calidad de vida de pacientes con cardiodesfibrilador se puede afectar por ansiedad y depresión(3,8). En Colombia, un estudio con este mismo tipo de pacientes reporta mayor afectación de las dimensiones físicas y cambio en el estado de salud(9).

En síntesis, los cardiodesfibriladores han permitido preservar más vidas, pero se destaca la necesidad de estudiar la calidad de vida en todas sus dimensiones, en especial en la psicológica(10), en grupos de personas que conviven con dispositivos cardiacos para contribuir a su cuidado con herramientas científicas efectivas que mejoren el bienestar biopsicosocial(2). Así pues, el presente estudio tiene como objetivo describir la relación que existe entre la calidad de vida relacionada con la salud, la ansiedad y la depresión en personas portadoras de un cardiodesfibrilador.

\section{Materiales y métodos}

Estudio es de tipo cuantitativo, descriptivo correlacional, de corte transversal, con una muestra no probabilística de 85 pacientes de un Hospital Universitario en la ciudad de Bucaramanga, los cuales cumplieron los criterios de inclusión: hombres y mujeres con cardiodesfibrilador unicameral o bicameral, mayores de 18 años con estado de conciencia y orientación conservados, sin dificultades en el lenguaje oral y auditivo, con máximo tres años de convivencia con el dispositivo.

Se consideraron como criterios de exclusión la ubicación geográfica de difícil acceso. Para la recolección de datos se emplearon tres instrumentos pre-validados que permiten medir en la muestra seleccionada la calidad de vida relacionada con la salud, la ansiedad y depresión descritas en el siguiente apartado.

\section{Instrumentos}

Para medir la variable Calidad de vida relacionada con la salud, se empleó el instrumento Short-Form Health Survey (SF-36), desarrollado por Ware y Sherbourne en 1992. El SF-36, en su idioma original, cuenta con confiabilidad y validez de apariencia, de contenido, de criterio (concurrente y predictiva) y de constructo. En Colombia, la confiabilidad del instrumento arrojó un Alfa de Cronbach mayor de 0,80 . Se aplica a personas mayores de 18 años, con cualquier grado de escolaridad, mediante entrevista o autodiligenciamiento asistido(11). El inventario se compone de 36 ítems y nueve dimensiones, con puntajes de mínimo 0 y máximo 100 , lo que demuestra que a mayor valor de esta sumatoria, mayor es la calidad de vida en relación con los problemas de salud.

Para la variable Ansiedad, se empleó la Escala de Beck, desarrollada por Aaron Beck, et al., en 1988(12). Se eligió la versión mexicana, que cuenta con pruebas de validez adecuada y Alfa de Conbrach de 0,84 y $0,83(13)$. El instrumento contiene 21 ítems (escala tipo Likert 0-3) y la interpretación de la ansiedad varía de acuerdo a la puntuación: 
mínima $=0-5$, leve $=6-15$, moderada $=16-30$ y severa $=$ 31-63.

Para la variable Depresión, se empleó el Inventario de depresión de Beck (Beck Depression Inventory BDI)(12), que cuenta con 21 ítems (escala tipo Likert 0 -3). La puntuación de las opciones va aumentando de acuerdo con la severidad de los síntomas de depresión; la interpretación se realiza de la siguiente manera: mínima=0-9, media $=10-16$, moderada $=17$ 29 y severa=0-63. En México, se demostró la confiabilidad (con un Alfa de Cronbach de 0,87), la validez de contenido, la validez de constructo (tres factores principales, rotación Varimax) y la validez concurrente del instrumento(14).

Durante el proceso de la recolección de la muestra se encontraron limitaciones en el estudio, tales como el difícil acceso geográfico que impidió la asistencia de las personas al centro hospitalario, la desactualización en la base de datos institucional (direcciones, contactos telefónicos propios y de familiares), dificultando la captación de la muestra.

Se contactó a los pacientes por vía telefónica. Posteriormente, se solicitó el consentimiento informado, se aplicó una ficha sociodemográfica, el instrumento SF-36 versión 2, y los Inventarios de ansiedad y depresión de Beck. Para el procesamiento de los datos obtenidos, se construyeron dos bases de datos en el paquete estadístico SPSS 22.0.

\section{Consideraciones éticas}

La participación de los pacientes fue voluntaria, previa explicación de los alcances de la investigación. El estudio contó con la aprobación del Comité de Ética de la Facultad de Enfermería de la Universidad Nacional de Colombia. Una vez aprobado, se presentó el proyecto ante el Comité de Ética de Los Comuneros, Hospital Universitario de Bucaramanga.

\section{Resultados}

Entre las variables sociodemográficas recolectadas, se identificó un rango de edad de 36-92 años, con una media de 65 años de edad $(\mathrm{DE}= \pm 10,5)$. Los pacientes se encontraban con un tiempo de inserción del cardiodesfibrilador como mínimo de un mes y máximo de 35 meses, con media de 15,9 meses. En la Tabla 1 se describe la distribución de los pacientes según género, estado civil y convivencia.
En cuanto a las variables sociodemográficas relacionadas con la salud, todos los participantes tenían afiliación a la seguridad social. El rango de tiempo de permanencia con dispositivo más significativo fue de 1 a 2 años; la fracción de eyección del ventrículo izquierdo al momento del implante del dispositivo fue menor al $40 \%$ y un $24,7 \%$ había experimentado descargas del dispositivo.

Tabla 1. Frecuencias de las variables sociodemográficas $(n=85)$

\begin{tabular}{lrr}
\hline \multicolumn{3}{c}{ Variables sociodemográficas } \\
\hline \multicolumn{1}{c}{ Variable } & Frecuencia (n) & \% \\
\hline Género & 69 & 81,2 \\
Masculino & 16 & 18,8 \\
Femenino & & \\
Estado civil & 47 & 55,3 \\
Casado/a & 12 & 14,1 \\
Soltero/a & 9 & 10,6 \\
Viudo/a & 13 & 15,3 \\
Unión libre & 4 & 4,7 \\
Divorciado/a & & \\
¿Con quién vive? & 15 & 17,6 \\
Solo/a & 15 & 17,6 \\
Esposo/a & 46 & 54,2 \\
Esposo/a-hijos & 9 & 10,6 \\
Nuera/yerno-hijos & \multicolumn{2}{c}{} \\
& &
\end{tabular}

En relación a la variable Calidad de vida relacionada con la salud, esta presentó una distribución normal. El valor mínimo reportado en el estudio fue de 15,07 y el máximo, de 90,9, con una media de 59,7 $(\mathrm{DE}= \pm 19,16)$. La mayoría de los participantes de la muestra $(75,3 \%)$ percibió su estado de salud mejor al que tenían hace un año. En la Tabla 2 se describen todas las dimensiones evaluadas en la calidad de vida relacionada con la salud, siendo la más afectada el Desempeño físico.

Tabla 2. Descripción de las medidas de las dimensiones de calidad de vida relacionadas con la salud $(n=85)$

\begin{tabular}{lrrr}
\hline \multicolumn{1}{c}{ Dimensión } & \multicolumn{1}{c}{ Media (DE) } & Mínimo & Máximo \\
\hline Función física & $57,82(25,19)$ & 0 & 100 \\
Desempeño físico & $38,1(29,31)$ & 0 & 100 \\
Dolor corporal & $59,99(30,29)$ & 11,11 & 100 \\
Salud general & $58,0(15,96)$ & 5 & 100 \\
Vitalidad & $63,82(23,34)$ & 0 & 100 \\
Función social & $70,72(32,70)$ & 0 & 100 \\
Desempeño & $58,23(34,53)$ & 0 & 100 \\
emocional & $73,74(21,07)$ & 5 & 100 \\
Salud mental & & & \\
\hline
\end{tabular}


La variable Ansiedad presentó una distribución normal. La media que se obtuvo fue de 15,42 (DE= $\pm 10,31)$. El valor mínimo reportado fue de 0 y el máximo de 41. El nivel de ansiedad en personas portadoras de cardiodesfibrilador fue severo para el $8,2 \%$, moderado para $36,5 \%$, leve para el $37,6 \%$ y mínimo para el 17,6\%, lo que indica bajos niveles. Cabe resaltar que todos los pacientes presentaron por lo menos algún grado de ansiedad.

La variable Depresión presentó una distribución anormal. La media que se obtuvo fue de 10,33 $(\mathrm{DE}= \pm 8,57)$. El valor mínimo reportado fue de $0 \mathrm{y}$ el máximo de 41. El nivel de depresión en personas portadoras de cardiodesfibrilador fue mínimo para el $57,6 \%$ (la mayoría), medio para el 22,4\% y moderada para el 15,3\%; mientras que únicamente el 4,7\% presentó un grado severo de depresión. Sin embargo, al realizar la sumatoria de porcentajes, se evidenció que el $42,4 \%$ presentó niveles de depresión media, moderada y severa.
Relación entre la calidad de vida relacionada con la salud, la ansiedad y la depresión de las personas portadoras de cardiodesfibrilador. A partir de la correlación entre Calidad de vida relacionada con la salud y las variables Ansiedad y Depresión, se halló que la mayor relación negativa la presentó Ansiedad ( $r s=-0,609 ; p<0,0001)$.

Las variables Ansiedad y Depresión mostraron una relación positiva moderada con significancia estadística $(r s=0,446 ; p<0,0001)$. La Tabla 3, muestra algunas correlaciones entre las dimensiones de calidad de vida relacionada con la salud y las variables ansiedad y depresión.

Por último, se realizó un modelo de regresión lineal múltiple entre Calidad de vida relacionada con la salud, Ansiedad y Depresión, encontrando que la ansiedad y la depresión afectaron negativamente la calidad de vida relacionada con la salud (Ansiedad: $\beta=-0,844 ; p<0,0001$; Depresión: $\beta=-0,891 ; p<0,0001$ ), como se puede observar en la Tabla 4 .

Tabla 3. Correlaciones de las dimensiones Calidad de vida relacionada con la salud con las variables Ansiedad y Depresión en personas portadoras de un cardiodesfibriladores $(n=85)$

\begin{tabular}{|c|c|c|c|}
\hline Dimensión de calidad de vida & \multicolumn{2}{|c|}{ Coeficiente de correlación - Ansiedad } & Coeficiente de correlación - Depresión \\
\hline \multirow[t]{2}{*}{ Calidad de vida global } & & Pearson $r=-0,622$ & Spearman $r=-0,599^{* *}$ \\
\hline & & $p=0$ & $p=0$ \\
\hline \multirow[t]{2}{*}{ Funcionamiento físico } & & Spearman $r s=-0,416$ & Spearman $r s=-0,420$ \\
\hline & & $p=0$ & $p=0$ \\
\hline \multirow[t]{2}{*}{ Desempeño físico } & & Spearman $r s=-0,434$ & Spearman $r s=-0,487$ \\
\hline & & $p=0$ & $p=0$ \\
\hline \multirow{2}{*}{ Dolor corporal } & & Spearman $r s=-0,257$ & Spearman $r s=-0,222^{*}$ \\
\hline & & $p=0,017$ & $p=0,021$ \\
\hline \multirow[t]{2}{*}{ Salud general } & & Spearman $r s=-0,384$ & Spearman $r s=-0,434$ \\
\hline & & $p=0$ & $p=0$ \\
\hline \multirow[t]{2}{*}{ Vitalidad } & & Pearson $r=-0,604^{*}$ & Spearman $r s=-0,568$ \\
\hline & & $p=0$ & $p=0$ \\
\hline \multirow[t]{2}{*}{ Funcionamiento social } & & Spearman $r s=-0,527$ & Spearman $r s=-0,524$ \\
\hline & & $p=0$ & $p=0$ \\
\hline \multirow[t]{2}{*}{ Desempeño emocional } & & Spearman $r s=-0,405$ & Spearman $r s=-0,367^{* *}$ \\
\hline & & $p=0$ & $p=0$ \\
\hline \multirow[t]{2}{*}{ Salud mental } & & Spearman $r s=-0,569$ & Spearman $r s=-0,561$ \\
\hline & & $p=0$ & $p=0$ \\
\hline \multicolumn{4}{|c|}{$\begin{array}{l}\text { *La correlación es significativa en el nivel 0,01 ( } 2 \text { colas) } \\
\text { ** La correlación es significativa en el nivel } 0,01 \text { ( } 2 \text { colas })\end{array}$} \\
\hline Variables & Beta & Error estándar & $r$ cuadrado \\
\hline Constante & 81,923 & 29,240 & $<0,0001$ \\
\hline Total depresión & $-0,891$ & $-4,714$ & $<0,0001$ \\
\hline Total ansiedad & $-0,844$ & $-5,370$ & $<0,0001$ \\
\hline
\end{tabular}

Variable dependiente: Total calidad de vida (estandarizada) 


\section{Discusión}

En las variables sociodemográficas de este estudio se resalta la predominancia del género masculino de la muestra $(81,2 \%)$, información similar a la de una investigación en paises de América del Norte, Europa y Asia con el 79\%(15). Hallazgos similares se observaron en estudios llevados a cabo en China(8), Japón(16), Irán(17), Israel(18), México(3), Brasil(19), Estados Unidos(20,21), Australia(21), España(22,23), Alemania(24,25), Países Bajos(26,27), Dinamarca(28-30) y Suecia(31-33). No obstante, en otras publicaciones, poco más del $60 \%$ de los participantes correspondían al género masculino(34-36), mientras que en otras eran cerca del $68,4 \%$ de población masculina(17).

En relación con la edad, la media en este estudio fue de 65 años en un rango de 36-92 años, lo cual corresponde al incremento de la población adulta a la que se le implantan cardiodesfibriladores. Estos datos concuerdan con los resultados de estudios que describieron edades medias entre los 60 a 70 años $(8,15,18,20-26,30-33)$ con rangos extensos entre 17 a 94 años $(8,16,22,23,31,33,35)$. Otros estudios en países norteamericanos, europeos y oceánicos también han encontrado edades medias inferiores a los 60 años(3,17,19,27-29,35,36).

El 55,3\% de los usuarios estaban casados(as), información semejante a la del estudio de Hammash et al.,(21) y Berg et al.,(30), donde el 63\% y el 65\% tenían pareja, respectivamente, pero que difiere de otras investigaciones que han reportado valores cercanos o superiores al 70\%(3,8,17,26,29,36). Así mismo, el $54,1 \%$ de los pacientes participantes convivían con su esposo(a) e hijos, y en otras investigaciones un mayor porcentaje de usuarios vivían con alguien(8,16,19,22,24,25,31-34,37), esto explica que la familia participa en las actuaciones después del alta hospitalaria para la recuperación física y la reincorporación social de los pacientes portadores de cardiodesfibriladores.

El tiempo desde el implante del cardiodesfibrilador fue de 15,98 meses (DE= \pm 9 ), con un rango de 1-35 meses, aunque los hallazgos de otros autores arrojaron una media de 2,54 años (DE= $\pm 1,43)^{(8)}, 3,65$ años $(\mathrm{DE}= \pm 3,4)^{(38)}, 4,4$ años $(\mathrm{DE}= \pm 3,8)^{(18), 4,7}$ años $(\mathrm{DE}= \pm 3,9)^{(21,22,33)}, 5,3$ años $(\mathrm{DE}= \pm 3,7)^{(24,25)}$ y 5,7 $(\mathrm{DE}=4,1)^{(32)}$ demostrando mayor tiempo transcurrido desde la inserción del dispositivo.
Por otra parte, se observó que el $24,7 \%$ de los participantes habían experimentado descargas del dispositivo, resultados similares a los obtenidos en una investigación de Dinamarca con un 24\%(37). Los valores difieren de los resultados en Suecia, España, Australia, Estados Unidos y Brasil, cuyos porcentaje de usuarios con historiales de descarga fueron superiores al 35\% pero inferiores al 50\%(16,19,21,22,3133), y del mismo modo, en algunos países europeos y asiáticos donde menos del $14 \%$ de participantes experimentaron descargas $(15,26,28,38)$. Esto muestra una variabilidad del historial de descargas en los participantes de diferentes estudios.

Maron et al.,(35) estudiaron el tipo descargas experimentadas en los pacientes, y encontraron que en Estados Unidos, Europa y Australia las descargas apropiadas fueron del 19\% e inadecuadas del 20\%, mientras que Varghese et al.,(25) encontraron que en Alemania tanto las descargas adecuadas e inadecuadas eran del 9\%, además de ser una de las primeras complicaciones experimentadas después de la primera implantación del dispositivo.

A pesar de que el presente estudio no diferenció el tipo de descargas experimentadas por los usuarios, los porcentajes de estos estudios nombrados previamente son menores a lo encontrado. Cabe resaltar, que las descargas del dispositivo se han asociado a una disminución de la calidad de vida(15) con una reducción en la mejoría de los síntomas de depresión y ansiedad(28), o un aumento de los puntajes de ansiedad(15). En Alemania, autores concluyeron que los niveles de ansiedad son más altos como consecuencia del fenómeno del shock fantasma ${ }^{(24)}$. Siendo este definido por primera vez en 1992 como aquel shock referido, en ocasiones vívidamente experimentado, por el paciente y no registrado por el dispositivo(39).

La media de la calidad de vida relacionada con la salud en el presente estudio fue de 59,7 $(\mathrm{DE}= \pm 19,16)$. Otra investigación utilizó también un instrumento con puntuaciones de 0 a 100 (de peor a mejor calidad de vida), denominado el Kansas City Cardiomyopathy Questionnaire (KCCQ), encontrando una media de 76,7 puntos $(\mathrm{DE}= \pm 14,68)$ en una muestra más pequeña (42 pacientes) ${ }^{(3)}$.

Otros autores además han utilizado el Minnesota Living with Heart Failure Questionnaire (MLHFQ), un instrumento con puntajes entre 0 a 105 (de mejor a 
peor calidad de vida) y han encontrado una media cercana a los 30 puntos(24,25). La mayoría de los estudios han utilizado en sus mediciones de calidad de vida las diferentes versiones de la EuroQol - 5D (EQ - 5D), un instrumento que evalúa el sistema descriptivo de $-0,59$ a 1,0 (de peor a mejor calidad de vida) y la escala analógica visual de 0 a 100 (de peor a mejor calidad de vida), y las medias de los puntajes obtenidos se inclinan hacia una mejor calidad de vida al ser cercanas o mayores a 0,7 y $75^{(21,30-33)}$. Los resultados de estos estudios señalan una mejor calidad de vida con respecto a la encontrada en este estudio.

No obstante, la media de calidad de vida es similar a los datos que reportan autores al utilizar la HeartQoL, donde se halló un valor de 1,66 $(\mathrm{DE}= \pm 0,78)$, siendo posibles los rangos entre 0 a 3 (de peor a mejor calidad de vida)(30); o con la escala análoga de las versiones de EQ - 5D, con medias cercanas a 65(15,30). Cabe resaltar que otras investigaciones han utilizado la SF12 v.2 Health Survey relacionando el bienestar con la calidad de $\operatorname{vida}^{(20,35)}$ y algunos autores, refieren que en la mayoría de los pacientes la calidad de vida mejora después del procedimiento de implantación(22,34,36), o no se altera ${ }^{(35)}$. Sin embargo, este estudio encontró que la mayoría de usuarios participantes $(75,3 \%)$ identifican una evolución reciente sobre un mejor estado de salud.

Con respecto a las dimensiones de la calidad de vida, se encontró que la dimensión Salud mental reveló una relación débil positiva con significancia estadística del historial de descargas $(r s=0,238$; $p=0,028)$, es decir, a medida que aumenta el historial de descargas la salud mental se afecta.

Otros estudios muestran, que en pacientes del primer año del implante, la dimensión Salud mental empeoraba con los choques, así como las dimensiones Función física, Salud general y Rol emocional(23). Por su parte, autores que han utilizado la SF12 v.2 Health Survey encontraron que no hay una diferencia significativa entre los usuarios que presentan y no presentan descargas $(50,5$ puntos; $\mathrm{DE}= \pm 10,2$ vs. 52,0 puntos; $\mathrm{DE}= \pm 8,4$ ) en el componente de bienestar mental de la escala(35).

Las dimensiones con puntajes más altos (y por ende mejor conservadas), correspondieron a Salud mental $(73,74 ; \quad D E= \pm 21,07)$, Función social $\quad(70,72$;
$\mathrm{DE}= \pm 32,70)$ y Vitalidad $(63,82 ; \mathrm{DE}= \pm 23,34)$. Estos resultados concuerdan con van Veen et al.,(29) donde la dimensión Salud mental $(70,9 ; \quad D E= \pm 19,2)$ y Función social $(65,2 ; \mathrm{DE}= \pm 28,7)$ tenían los mejores puntajes; pero Dolor corporal $(66,0$; $\mathrm{DE}= \pm 27,7)$ tuvo una puntuación más alta que Vitalidad $(56,0$; $\mathrm{DE}= \pm 21,9$ ). Mientras que Wong et al.,(8) encontraron puntajes más altos en Desempeño emocional (90,1; $\mathrm{DE}= \pm 22,2) \quad y$ Dolor corporal $(85,0 ; \quad \mathrm{DE}= \pm 23,6)$, aunque coinciden con altos puntajes en Función social $(89,7 ; \quad D E= \pm 22,7)$ y Salud mental $(77,3$; $\mathrm{DE}= \pm 21,4)$. Magnusson et al.,(37) coinciden con altos resultados en Salud mental $(71,8 ; \mathrm{DE}= \pm 22,9)$ y Función social $(75,1 ; \mathrm{DE}= \pm 26,2)$.

En la investigación de Cesarino et al.,(36) en cambio se coincide únicamente con la dimensión de Función social $(80,5 ; D E= \pm 26,9)$, puesto que las otras dimensiones mejor conservadas son Salud general $(67,3 ; \mathrm{DE}= \pm 24,6)$ y Dolor corporal $(66,6 ; \mathrm{DE}= \pm 29,1)$, aunque los puntajes de las dimensiones Vitalidad $(64,2 ; \mathrm{DE}= \pm 26)$ y Salud mental $(64,1 ; \mathrm{DE}= \pm 27,4)$ no difieren por mucho de estas dos últimas. En general los estudios mencionados coindicen con Función social dentro de las tres primeras dimensiones mejor conservadas.

La dimensión de calidad de vida relacionada con la salud más afectada en el presente estudio fue Desempeño físico, con una media de 38,1 ( $D E= \pm 29,31$ ), lo que coincide con los hallazgos del estudio de van Veen et al. $(35,4 ; \mathrm{DE}= \pm 40,2)^{(29)}$. No obstante, difiere con Wong et al.,(8) Magnusson et al.(37) y Cesarino et al.,(36) que reportan una media en esta dimensión más alta $(69,5 ; \mathrm{DE}= \pm 36,5 ; 57,4$; $\mathrm{DE}= \pm 43,6 ; 54,1 ; \mathrm{DE}= \pm 23,8$, respectivamente) que supone una mayor conservación. Para estos autores, Salud general(8), Vitalidad(8,37) y Función física(36) fueron las dimensiones con unos de los puntajes más bajos.

En el presente estudio, los niveles de ansiedad severa y mínima representaron una pequeña proporción $(8,2 \%$ y $17,6 \%$, respectivamente), mientras que la mayoría de los pacientes presentaron ansiedad leve $(37,6 \%)$ o moderada (36,5\%). Varios estudios reportan la aplicación del instrumento Hospital Anxiety and Depression Scale (HADS-A), y con respecto a los niveles encontrados, en Irán y México se han encontrado niveles inferiores de ansiedad leve-moderada $(24,2 \%$ y 
$40,5 \%$, respectivamente) y mayores de ansiedad severa $(26,3 \% \text { y } 11,9 \% \text {, respectivamente })^{(3,17)}$; mientras que en Dinamarca tanto los niveles de ansiedad leve $(17,8 \%)$ y moderada-severa $(11,9 \%)$ son menores(30).

Los usuarios que tuvieron niveles de ansiedad levemoderada-severa fueron el $82,3 \%$, que difieren de los estudios con la HADS-A de Suecia y China donde apenas fueron el 15,5\%(32), 15,9\%(33) y el $14,4 \%{ }^{(8)}$; de Estados Unidos y Australia, con el 25\%(21); y de publicaciones previamente nombradas en México, Dinamarca e Irán, donde los valores están entre el $30 \%$ y $55 \%{ }^{(3,17,30)}$.

La media identificada para la variable Ansiedad fue de 15,42 (DE= $\pm 10,31)$, señalando un nivel moderado. La mayoría de autores han utilizado el HADS-A, y a diferencia de este estudio, han encontrado puntuaciones que se consideran normales al estar entre 0 a 7: en Estados Unidos y Australia reportaron una media de $5(\mathrm{DE}= \pm 3,8)^{(21)}$; en Alemania de 3,88 $(\mathrm{DE}= \pm 3,8)^{(24)}$ y $3,9(\mathrm{DE}= \pm 3,8)^{(25)}$; en Países Bajos de $5,13 \quad(\mathrm{DE}= \pm 3,72)^{(27)}$; en Dinamarca de 3,72 $(\mathrm{DE}= \pm 3,58)^{(28)}$ y $5,5(\mathrm{DE}= \pm 4,2)^{(30)}$; en Suecia de 3,8 $(\mathrm{DE}= \pm 3,7)^{(31)}$ y $3,60(\mathrm{DE}= \pm 3,58)^{(32)}$; y en China de 3,4 $(\mathrm{DE}= \pm 3,6)^{(38)}$.

En un estudio multicéntrico en Estados Unidos, Europa y Australia que comparó los puntajes de la HADS-A en usuarios con implante que habían experimentado descargas, $5,2 \quad(\mathrm{DE}= \pm 3,7)$, con aquellos que no, $5,5(\mathrm{DE}= \pm 3,9)^{(35)}$, tampoco se encontraron niveles que indicaran ansiedad, como si se encontró en el presente estudio.

Otra escala utilizada para medir la ansiedad, pero en menor cantidad, fue la State-Trait Anxiety Inventory (STAI), con la que se han identificado puntajes medios de 37,23 (DE= $\pm 11,46)$, que al ser menores a 40 no indican niveles clínicos probables de ansiedad. Cabe señalar que usando esta escala, una investigación estadounidense encontró ansiedad moderada al momento del alta hospitalaria y hasta los seis meses de seguimiento en los usuarios participantes, nivel de ansiedad también encontrado en el presente estudio(27).

En esta muestra se registró que los niveles de depresión severa se encontraron en una pequeña proporción para el 4,7\% y moderada para el 15,3\% mientras la mayoría presentaron depresión mínima $(57,6 \%)$ y media $(22,4 \%)$.

En general los estudios han utilizado el componente de depresión de la HADS-D, y con respecto a los niveles encontrados en México se han reportado niveles similares de depresión leve-moderada $(40,5 \%)$ y menores de depresión severa $(16,7 \%)^{(3)}$; en tanto que en Dinamarca los niveles de depresión leve (11\%) y moderada-severa (8\%) fueron menores(30).

Otra escala que ha medido la depresión, es la Hamilton Rating Scale for Depression (HAM-D), con la que se han obtenido niveles cercanos a la depresión media (19\%) obtenida, aunque inferiores al de moderada-severa (9\%).

Los participantes que informaron niveles de depresión en este estudio media-moderada-severa fueron el 42,4\%, que difieren de los estudios con la HADS-D de China y Suecia con porcentajes pequeños de $20,1 \%{ }^{(8)}, 7,4 \%^{(32)}$ y $8,5 \%{ }^{(33)}$; en Estados Unidos y Australia con el $18 \%{ }^{(21)}$; y del estudio nombrado previamente en Dinamarca(30), pero que es superado por el valor de la investigación en México(3).

De forma similar, con la HAM-D en Israel se obtuvo un $28 \%$ de usuarios que tenían depresión significativa(18), inferior a lo señalado en este estudio. Uno de los estudios que utilizó el mismo instrumento que en este investigación, halló un porcentaje menor de usuarios $(26,1 \%)$ con síntomas depresivos ${ }^{(16)}$.

La media identificada para la variable Depresión fue de 10,33 puntos $(\mathrm{DE}= \pm 8,57)$ e indica un nivel medio. La mayoría de autores han utilizado el HADS-D, y a diferencia de este estudio, han encontrado puntuaciones que se consideran normales al estar entre 0 a 7: en Estados Unidos y Australia reportaron una media de 4,3 (DE $= \pm 3,5)^{(21)}$; en Alemania 4,7 $(\mathrm{DE}= \pm 4,0)^{(25)}$ y $3,85(\mathrm{DE}= \pm 4,0)^{(24)}$; en Suecia de 2,66 $(\mathrm{DE}= \pm 2,79)^{(32)}$ y de $2,9(\mathrm{DE}= \pm 2,9)^{(31)}$; en Dinamarca de $3,80(\mathrm{DE}= \pm 3,81)^{(28)}$ y de $4,5(\mathrm{DE}= \pm 3,8)^{(30)}$; y en Japón una media de 4,2 puntos $(\mathrm{DE}= \pm 3,9)^{(38)}$.

En el estudio multicéntrico en Estados Unidos, Europa y Australia mencionado anteriormente, se halló con la HADS-D un puntaje de $3,2(\mathrm{DE}= \pm 2,7)$ para usuarios que habían experimentado descargas, y de $3,5(\mathrm{DE}= \pm 3,7)$ para aquellos que no, por lo que tampoco se encontraron niveles que indicaran 
depresión clínica(35), como si se hallaron en este estudio.

Otra escala utilizada pero en menor cantidad ha sido la Center for Epidemiologic Studies Depression (CESD), que encontró puntajes inferiores a 16 desde el momento del alta hasta el año de seguimiento, concluyendo menor riesgo de depresión(20).

Se evidenció una correlación negativa media estadísticamente significativa entre Calidad de vida relacionada con la salud y Ansiedad $(r=-0,622$; $p=<0,001$ ), lo que demuestra que, a mayor ansiedad, menor calidad de vida relacionada con la salud en personas portadoras de un cardiodesfibrilador. Este hallazgo lo confirman las investigaciones de Hammash et al.(21) y Figueroa-López et al.(3) que concluyeron que los niveles de ansiedad $(\beta=-0,18$, $p<0,05 ;$ y $r=-0,322, p<0,05$, respectivamente) se asociaron con niveles más bajos de calidad de vida relacionada con la salud.

Respecto a Depresión, en esta investigación se observó que a medida que ésta aumentaba, la calidad de vida disminuía $(r s=-0,599 ; p=0,001)$, resultados también encontrados por otros autores que asociaron fuertemente la depresión con niveles más bajos de calidad de vida(21). Sin embargo, difiere de los resultados del estudio realizado en población mexicana portadora de cardiodesfibrilador, en la que se encontró una relación débil no significativa estadísticamente $(r=-0,20 ; p=0,223)$. La depresión a su vez, se relacionó positivamente con la ansiedad $(r=0,305 ; p<0,05)$ de tal modo que a medida que los niveles de depresión aumentan también lo hacen los niveles de ansiedad( $(3)$.

A partir del modelo de regresión lineal múltiple, se observó que la ansiedad y la depresión afectan negativamente la calidad de vida relacionada con la salud (Ansiedad: $\beta=-0,844 ; p<0,0001$; Depresión: $\beta=-$ $0,891 ; \quad p=0,001)$. Algunos estudios sustentan parcialmente los resultados que arrojó esta investigación, como el de Elosegui(23), que por medio de coeficientes de correlación encontraron una asociación significativa entre Calidad de vida $y$ Ansiedad $(0,216)$ y Calidad de vida y Depresión $(0,329)$.

Asimismo, en otra investigación se encontró que la depresión y la ansiedad son factores asociados negativamente con la calidad de vida relacionada con la salud; la calidad de vida física se asoció con la depresión $(\beta=-1,61 ; \mathrm{DE}= \pm 0,24 ; p<0,001)$, mientras que la calidad de vida mental se asoció con la depresión $(\beta=-1,17 ; \quad \mathrm{DE}= \pm 0,19 ; \quad p<0,001) \quad$ y la ansiedad $(\beta=-1,12 ; \mathrm{DE}= \pm 0,20 ; p<0,001)^{(8)}$.

Finalmente, es importante destacar que esta alternativa de solución como lo es la implantación de un cardiodesfibrilador en pacientes con enfermedad isquémica, puede generar la disminución de la calidad de vida relacionada con la salud, especialmente en las dimensiones de función física, vitalidad y salud mental, esto se presenta por problemas alternos de depresión y ansiedad que padece el paciente como consecuencia del tratamiento en sí. Lo anterior, demuestra como un tratamiento que ofrece alternativa de vida a una persona, puede generar otros problemas que afectan la salud del paciente( $(4-6,8,10)$.

\section{Conclusiones}

Los resultados obtenidos en este estudio reportan mayor número de hombres como portadores de cardiodesfibrilador, con rango de edad amplio que va desde los 36-92 años, con afiliación social, tiempo de permanencia del dispositivo de uno a dos años, casados, con una fracción de eyección del ventrículo izquierdo menor del $40 \%$ anterior al implante y un nivel educativo básico.

Los pacientes reportaron disminución en la calidad de vida relacionada con la salud, presencia de ansiedad leve-moderada y algunos presentaron diferentes niveles de depresión. Esta investigación encontró relación entre la calidad de vida relacionada con la salud y la ansiedad y depresión desarrollada en los pacientes por el tratamiento en sí.

Ser portador de un cardiodesfibrilador desencadena ansiedad y depresión, en la medida que estos problemas aumentan, la calidad de vida se puede deteriorar, por lo tanto, la relación de estas variables, evidencian la necesidad de incluirlas como aspectos fundamentales en la valoración de enfermería y de otros miembros del equipo interdisciplinario con el fin de detectarlas de manera temprana e implementar intervenciones que propendan por el bienestar del paciente. 
Se recomienda desarrollar réplicas que permitan ampliar el análisis de las variables sociodemográficas y clínicas con la calidad de vida relacionada con la salud, ya que los pacientes con cardiopatías son más propensos a desencadenar diferentes niveles de Ansiedad y Depresión, cobra importancia realizar estudios comparativos en pacientes portadores y no portadores de cardiodesfibrilador.

Lo anterior, con el fin de evaluar nuevas relaciones que permitan comprender mejor los fenómenos asociados a esta población, y así procurar que el cuidado garantice la conservación de una calidad de vida relacionada con la salud de buen nivel. Es importante mencionar que el instrumento de ansiedad cuenta con preguntas que indagan sobre algunos de los síntomas del paciente cardiaco, lo cual puede dificultar la diferenciación entre la ansiedad y los síntomas propios del paciente en estudio.

\section{Agradecimientos}

A la Universidad Nacional de Colombia, Los Comuneros, Hospital Universitario de Bucaramanga, por su apoyo en el desarrollo de esta investigación y a los pacientes.

Conflictos de intereses: Ninguno declarado por las autoras.

\section{Referencias}

1. Organización Mundial de la Salud (OMS). Enfermedades cardiovasculares [Internet]. Centro de prensa. Ginebra: Organización Mundial de la Salud (OMS); 2015. Available from:

http://www.who.int/mediacentre/factsheets/fs317/es/

2. Ministerio de Salud, Instituto Nacional de Salud, Observatorio Nacional de Salud. Carga de enfermedad por enfermedades crónicas no transmisibles y discapacidad en Colombia [Internet]. 5ta ed. Bogotá D.C: Imprenta Nacional de Colombia; 2015. 1-212 p. Available from: https://www.minsalud.gov.co/sites/rid/Lists/BibliotecaDi gital/RIDE/IA/INS/informe-ons-5.pdf

3. Figueroa-López C, Rocha-Rodríguez V, Cigarroa-López Á, Ramos-Del Río B. Comorbilidad emocional y la aceptación psicológica al desfibrilador automático implantable. Rev Mex Cardiol [Internet]. 2016;27(S1):S4-12. Available from: https://www.medigraphic.com/pdfs/cardio/h2016/hs161a.pdf

4. Vázquez Ruiz de Castroviejo E, Muñoz Bellido J, Lozano Cabezas C, Ramírez Moreno A, Guzmán Herrera M, Tarabini Castellani A, et al. Análisis de la frecuencia de las arritmias cardíacas y de los trastornos de conducción desde una perspectiva asistencial. Rev Esp Cardiol [Internet]. 2005;58(6):657-65. Available from: https://doi.org/10.1157/13076418
5. Borleffs CJW, Van Erven L, Van Bommel RJ, Van Der Velde ET, Van Der Wall EE, Bax JJ, et al. Risk of failure of transvenous implantable cardioverter-defibrillator leads. Circ Arrhythmia Electrophysiol [Internet]. 2009;2(4):411-6. Available from: https://doi.org/10.1161/CIRCEP.108.834093

6. Dorantes Sánchez M. Complicaciones del cardioversordesfibrilador automático implantable. Tormenta eléctrica arrítmica. Rev Cuba Investig Biomed [Internet]. 2011;30(4):537-54. Available from: http://scielo.sld.cu/scielo.php?script=sci_arttext\&pid=S086 4-03002011000400011

7. Rodríguez-Morales MM, García-Niebla J, Valle-Racero JI. Intervenciones de enfermería al paciente portador de desfibrilador automático implantable. Cardiocore [Internet]. 2013;48(4):166-9. Available from: https://doi.org/10.1016/j.carcor.2012.10.005

8. Wong FMF, Sit JWH, Wong EML, Choi KC. Factors associated with health-related quality of life among patients with implantable cardioverter defibrillator: Identification of foci for nursing intervention. J Adv Nurs [Internet]. 2014;70(12):2821-34. Available from: https://doi.org/10.1111/jan.12434

9. Zuluaga Álzate LJ. Capacidad de agencia de autocuidado y calidad de vida de las personas que tienen dispositivos cardiacos implantables [Internet]. Universidad Nacional de Colombia; 2013:213. Available from: https://repositorio.unal.edu.co/handle/unal/49583

10. Cevik C, Perez-Verdia A, Nugent K. Implantable cardioverter defibrillators and their role in heart failure progression. Europace [Internet]. 2009;11(6):710-5. Available from: https://doi.org/10.1093/europace/eup091

11. Lugo LE, Garcia HI, Gomez C. Confiabilidad del cuestionario de calidad de vida en salud SF-36 en Medellin, Colombia. Rev Fac Nac Salud Pública [Internet]. 2006;24(2):37-50. Available from: http://www.scielo.org.co/pdf/rfnsp/v24n2/v24n2a05.pdf

12. Beck AT, Clark DA. Anxiety and depression: An information processing perspective. Anxiety Res [Internet]. 1988;1(1):23-36. Available from: https://doi.org/10.1080/10615808808248218

13. Galindo Vázquez 0 , Rojas Castillo E, Meneses García A, Aguilar Ponce JL, Álvarez Avitia MA, Alvarado Aguilar S. Propiedades psicométricas del inventario de ansiedad de beck (BAI) en pacientes con cáncer. Psicooncología [Internet]. 2015;12(1):51-8. Available from: https://doi.org/10.5209/rev_PSIC.2015.v12.n1.48903

14. Jurado S, Villegas ME, Méndez L, Rodríguez F, Loperena V, Varela R. La estandarizacion del Inventario de Depresion de Beck para los residentes de la Ciudad de Mexico. Salud Ment [Internet]. 1998;21(3):26-31. Available from: http://www.revistasaludmental.mx/index.php/salud_ment al/article/view/706/705

15. Sears SF, Rosman L, Sasaki S, Kondo Y, Sterns LD, Schloss EJ, et al. Defibrillator shocks and their effect on objective and subjective patient outcomes: Results of the PainFree SST clinical trial. Hear Rhythm [Internet]. 2018;15(5):734-40. Available

from: https://doi.org/10.1016/j.hrthm.2017.12.026

16. Ichikura K, Kobayashi S, Matsuoka S, Suzuki T, Nishimura K, Shiga $\mathrm{T}$, et al. Avoidance behavior associated with depressive symptoms in patients with implantable cardioverter defibrillators. Int J Clin Heal Psychol [Internet]. 
2017;17(1):1-8.

Available

from: https://doi.org/10.1016/j.ijchp.2016.11.001

17. Mohammadi N, Askari S, Farahani M, Ghorbani A, Ghafarzadegan R. Assessment of anxiety level in patients receiving implantable cardioverter defibrillator. Crescent J Med Biol Sci [Internet]. 2019;6(1):13-7. Available from: http://www.cjmb.org/uploads/pdf/pdf_CJMB_276.pdf

18. Amiaz R, Asher E, Rozen G, Czerniak E, Glikson M, Amiaz R, et al. Do implantable cardioverter defibrillators contribute to new depression or anxiety symptoms? A retrospective study. Int J Psychiatry Clin Pract [Internet]. 2016;20(2):101-5. Available from: https://doi.org/10.3109/13651501.2016.1161055

19. Braun S, Krüger J, Souza EN, Rabelo ER. Quality of life of patients with implanted cardiac devices: a transversal study. Online braz j nurs [Internet]. 2012;11(3):778-88. Available from: https://doi.org/10.5935/16764285.20120051

20. Dougherty CM, Fairbanks AM, Eaton LH, Morrison ML, Kim MS, Thompson EA. Comparison of patient and partner quality of life and health outcomes in the first year after an implantable cardioverter defibrillator (ICD). J Behav Med [Internet]. 2016;39(1):94-106. Available from: https://doi.org/10.1007/s10865-015-9671-0

21. Hammash M, McEvedy SM, Wright J, Cameron J, Miller J, Ski $\mathrm{CF}$, et al. Perceived control and quality of life among recipients of implantable cardioverter defibrillator. Aust Crit Care [Internet]. 2019;32(5):383-90. Available from: https://doi.org/10.1016/j.aucc.2018.08.005

22. Thylén I, Dekker RL, Jaarsma T, Strömberg A, Moser DK. Characteristics associated with anxiety, depressive symptoms, and quality-of-life in a large cohort of implantable cardioverter defibrillator recipients. J Psychosom Res [Internet]. 2014;77(2):122-7. Available from: https://doi.org/10.1016/j.jpsychores.2014.05.007

23. Elosegui Itxaso L. Calidad de vida en pacientes con Desfibrilador Automático Implantable [Internet]. Tesis de doctorado; Universidad de Zaragoza. 2014. Available from: http://zaguan.unizar.es/record/15780/files/TESIS-2014074.pdf

24. Varghese S, Geller JC, Ohlow MA. Phantom shocks in implantable cardioverter-defibrillator recipients: impact of education level, anxiety, and depression. Herzschrittmachertherapie und Elektrophysiologie [Internet]. 2019;30(3):306-12. Available from: https://doi.org/10.1007/s00399-019-00645-y

25. Varghese S, Geller JC, Ohlow MA. Decision regret in implantable cardioverter-defibrillator recipients: A crosssectional analysis on patients that regret their decision after ICD implantation. Herzschrittmachertherapie und Elektrophysiologie [Internet]. 2020;31(1):77-83. Available from: https://doi.org/10.1007/s00399-020-00675-x

26. Pedersen SS, Andersen CM, Denollet J, Habibovic M. Depressive symptoms in patients with an implantable cardioverter defibrillator: Does treatment expectations play a role? Gen Hosp Psychiatry [Internet]. 2018;51(December 2017):10-4. Available from: https://doi.org/10.1016/j.genhosppsych.2017.12.002

27. Emons WH, Habibović M, Pedersen SS. Prevalence of anxiety in patients with an implantable cardioverter defibrillator: measurement equivalence of the HADS-A and the STAI-S. Qual Life Res [Internet]. 2019;28(11):3107-16. Available from: https://doi.org/10.1007/s11136-019-02237-2
28. Rottmann N, Skov O, Andersen CM, Theuns DAMJ, Pedersen SS. Psychological distress in patients with an implantable cardioverter defibrillator and their partners. J Psychosom Res [Internet]. 2018;113:16-21. Available from: https://doi.org/10.1016/j.jpsychores.2018.07.010

29. van Veen B, Andersen CM, Johansen JB, Theuns DA, Pedersen SS. Patient-Reported Quality of Life as a Predictor of Mortality and Ventricular Tachyarrhythmia's During 7 Years' Follow-Up in Patients With an Implantable Cardioverter Defibrillator (from the MIDAS Study). Am J Cardiol [Internet]. 2019;123(4):605-10. Available from: https://doi.org/10.1016/j.amjcard.2018.11.021

30. Berg SK, Rasmussen TB, Mols RE, Thorup CB, Borregaard B, Christensen $\mathrm{AV}$, et al. Both mental and physical health predicts one year mortality and readmissions in patients with implantable cardioverter defibrillators: findings from the national DenHeart study. Eur J Cardiovasc Nurs [Internet]. 2019;18(2):96-105. Available from: https://doi.org/10.1177/1474515118794598

31. Miller JL, Thylén I, Moser DK. Gender disparities in symptoms of anxiety, depression, and quality of life in defibrillator recipients. PACE [Internet]. 2016;39(2):14959. Available from: https://doi.org/10.1111/pace.12786

32. Israelsson J, Thylén I, Strömberg A, Bremer A, Årestedt K. Factors associated with health-related quality of life among cardiac arrest survivors treated with an implantable cardioverter-defibrillator. Resuscitation [Internet]. 2018;132:78-84. Available from: https://doi.org/10.1016/j.resuscitation.2018.09.002

33. Miller JL, Thylén I, Elayi SC, Etaee F, Fleming S, Czarapata $\mathrm{MM}$, et al. Multi-morbidity burden, psychological distress, and quality of life in implantable cardioverter defibrillator recipients: Results from a nationwide study. J Psychosom Res [Internet]. 2019;120(November 2018):39-45. Available from: https://doi.org/10.1016/j.jpsychores.2019.03.006

34. Haugaa KH, Potpara TS, Boveda S, Deharo J, Chen J, Dobreanu D, et al. Patients' knowledge and attitudes regarding living with implantable electronic devices: results of a multicentre, multinational patient survey conducted by the European Heart Rhythm Association. Europace [Internet]. 2018;20:386-91. Available from: https://doi.org/10.1093/europace/eux365

35. Maron BJ, Casey SA, Olivotto I, Sherrid MV, Semsarian C, Autore C, et al. Clinical Course and Quality of Life in HighRisk Patients with Hypertrophic Cardiomyopathy and Implantable Cardioverter-Defibrillators. Circ Arrhythmia Electrophysiol [Internet]. 2018;11(4):1-9. Available from: https://doi.org/10.1161/CIRCEP.117.005820

36. Cesarino CB, Beccaria LM, Aroni MM, Rodrigues LCC, Pacheco S da S. Qualidade de vida em pacientes com cardioversor desfibrilador implantável: utilização do questionário SF-36. Rev Bras Cir Cardiovasc [Internet]. 2011;26(2):238-43. Available from: https://doi.org/10.1590/S0102-76382011000200014

37. Magnusson P, Mörner S, Gadler F, Karlsson J. Health-related quality of life in hypertrophic cardiomyopathy patients with implantable defibrillators. Health Qual Life Outcomes [Internet]. 2016;14(62):1-9. Available from: https://doi.org/10.1186/s12955-016-0467-x

38. Wong FMF. Factors associated with anxiety and depression among patients with implantable cardioverter defibrillator. J Clin Nurs [Internet]. 2017;26(9-10):1328-37. Available from: https://doi.org/10.1111/jocn.13637 
39. Kowey PR, Marinchak RA, Rials SJ. Things that go bang in the night. N Engl J Med [Internet]. 1992;327(26):1884.
Available

https://doi.org/10.1056/NEJM199212243272614 from: 